\title{
ORDERINGS, MULTICOLORING, AND CONSISTENTLY ORDERED MATRICES*
}

\section{DAVID L. HARRAR II ${ }^{\dagger}$}

\begin{abstract}
The use of multicoloring as a means for the efficient implementation of diverse iterative methods for the solution of linear systems of equations, arising from the finite difference discretization of partial differential equations, on both parallel (concurrent) and vector computers has been extensive; these include SOR-type and preconditioned conjugate gradient methods as well as smoothing procedures for use in multigrid methods. Multicolor orderings, corresponding to reorderings of the points of the discretization, often allow a local decoupling of the unknowns. Some new theory is presented which allows one to quickly verify whether or not a member of a certain class of matrices is consistently ordered (or $\pi$-consistently ordered) solely by looking at the structure of the matrix under consideration. This theory allows one to quickly ascertain that, while many well-known multicoloring schemes do give rise to coefficient matrices which are consistently ordered, many others do not. Some alternative orderings and multicoloring schemes proposed in the literature are surveyed and the theory is applied to the resulting coefficient matrices.
\end{abstract}

Key words. multicoloring, consistently ordered matrices, iterative methods, concurrent computers

AMS(MOS) subject classifications. 15,65

1. Introduction. The discretization by finite differences, or finite elements, of elliptic partial differential equations often leads to the solution of linear systems of equations

$$
A u=f
$$

With the advent of parallel computers and vector processors, it has become apparent that the use of alternative orderings, i.e., other than the natural or lexicographic ordering, may increase efficiency in the implementation of many iterative methods for solving (1); these methods include the Jacobi, Gauss-Seidel, and successive overrelaxation (SOR) iterations, and various preconditioned conjugate gradient (PCG) methods, as well as smoothing procedures for use in multigrid methods.

This leads naturally to the use of the technique of multicoloring to decouple the unknowns at the grid points of a finite difference, or finite element, discretization of a partial differential equation. The basic idea is to "color" the grid points so that unknowns corresponding to grid points of a particular color are coupled only with unknowns of other colors. Thus all unknowns of a single color can be updated simultaneously, i.e., in parallel, or with a single vector instruction, assuming that the unknowns are stored appropriately.

In general, multicoloring with $p$ colors corresponds to a partitioning $\pi$ of the

* Received by the editors July 2, 1990; accepted for publication (in revised form) May 8, 1991. This research was supported in part by Department of Energy grant DE-FG03-89ER25073 and by the National Science Foundation under Cooperative Agreement CCR-8809615. The government has certain rights in this material.

$\dagger$ Department of Applied Mathematics 217-50, California Institute of Technology, Pasadena, California 91125 (dlh@ama.caltech.edu). 
coefficient matrix of the system (1) into the block $p \times p$ form

$$
A_{p}=\left[\begin{array}{ccccc}
A_{1,1} & A_{1,2} & \cdot & . & A_{1, p} \\
A_{2,1} & \cdot & \cdot & & \cdot \\
\cdot & \cdot & \cdot & \cdot & \cdot \\
\cdot & & \cdot & \cdot & A_{p-1, p} \\
A_{p, 1} & \cdot & \cdot & A_{p, p-1} & A_{p, p}
\end{array}\right],
$$

where the diagonal blocks $A_{i i}$ are square. Full decoupling of all unknowns of a given color from those of other colors obtains if the $A_{i i}$ are diagonal, and, in general, a significant number of the off-diagonal blocks $A_{i j}$ contain only zeros. Throughout this paper we maintain the notational convention that a single subscript on a matrix name is used to emphasize the block order of that matrix; when necessary for clarity, the corresponding partitioning $\pi$ carries the same subscript as in " $\pi_{p} . "$

Multicoloring has been used ubiquitously for the solution of linear systems by iterative methods on both parallel and vector computers (for a review, see, e.g., Ortega and Voigt [16]). Although more often a multicoloring scheme is used in conjunction with SOR-type iterative methods (e.g., Adams and Ortega [2] and O'Leary [15]), it can also prove useful with PCG methods. Poole and Ortega [18] use multicoloring to carry out incomplete Cholesky preconditioning on vector computers, and Harrar and Ortega [11] used a red/black ordering to efficiently vectorize a symmetric successive overrelaxation (SSOR) preconditioner. The parallel and vector implementation of SSOR PCG (as well as SOR) via multicoloring is also discussed in Harrar and Ortega [10], where a compromise is proposed between the faster convergence rate obtained with the natural ordering and the superior degree of parallelism and/or vectorization provided by the red/black ordering (see $§ 5.2$ ).

When solving elliptic problems using multigrid methods, much of the computation time is spent on the relaxation procedure used at each grid level. Multicoloring is useful in this area as well. For example, Gauss-Seidel smoothing with a red/black ordering is quite effective (Foerster, Stüben, and Trottenberg [5]), alternating direction line methods are particularly robust, and zebra orderings (§5.1) are useful for anisotropic equations (Stüben and Trottenberg [20]).

Not long ago a fair amount of attention was given to the concept of consistently ordered $(\mathrm{CO})$ matrices (see $\S 2$ ) and some generalizations thereof: generalized $\mathrm{CO}$ (GCO), $\mathrm{CO}(q, r)$ (see $\S 6), \operatorname{GCO}(q, r)$ (we note that GCO $(q, r)$ matrices are $p$-cyclic in the sense of Varga [23]), and $\pi$-CO matrices (Young [25]). Much of the foundation of the work done in this area can be found in the classical texts, Young [25] and Varga [23]. Lately, however, interest in whether or not the coefficient matrix $A$ of (1) is consistently ordered has somewhat waned. As a result, we often work with a system of equations that is not $\mathrm{CO}$ (or GCO, $\pi$-CO, etc.) when a simple permutation of the elements of $A$ might yield a matrix with one or more of these properties. The motivation for wanting the coefficient matrix $A$ to have one or more of these properties is discussed in $\S 2$ along with some concepts related to consistent ordering.

In $\S \S 3$ and 4 , we give some new theoretical results as to when matrices with a certain underlying block structure may be $\mathrm{CO}$ or $\pi$ - $\mathrm{CO}$ ("block" $\mathrm{CO}$ ). In $\S 5$, we apply these results to show the consistent ordering of some standard alternative orderings and the lack of this property for some other orderings proposed in the literature. Section 6 contains some applications of the results to another class of matrices, and in $\S 7$ we summarize our results. 
2. Consistently ordered matrices and related concepts. One property that may or may not obtain for the coefficient matrix $A$ as a result of a reordering of the unknowns is that of being a $C O$ matrix. Rather than appealing directly to the definition of a CO matrix (Young [25, Def. 5.3.2]), it is often more convenient to use the notion of a compatible ordering vector.

Definition 2.1. The vector $\gamma=\left(\gamma_{1}, \gamma_{2}, \ldots, \gamma_{n}\right)^{T}$, where the $\gamma_{i}$ are integers, is a compatible ordering vector for the matrix $A$ of order $n$ if, for $a_{i j} \neq 0$,

$$
\gamma_{i}-\gamma_{j}=\left\{\begin{aligned}
1 & \text { if } i>j \\
-1 & \text { if } i<j
\end{aligned}\right.
$$

The usefulness of compatible ordering vectors is made clear by the following theorem.

TheOREM 2.2 (Young [25, Thm. 5.3.2]). A matrix $A$ is consistently ordered if and only if a compatible ordering vector exists for $A$.

Determination of the optimum relaxation parameter for the SOR method applied to the system (1) via Young's classical SOR theory [25] is based upon the relationship $(\lambda+\omega-1)^{2}=\lambda \omega^{2} \mu^{2}$ between the eigenvalues $\mu$ and $\lambda$ of the Jacobi and SOR iteration matrices, respectively, associated with $A ; \omega$ is the relaxation parameter. The derivation of this eigenvalue relation is based upon a determinantal invariance which is true for $T$-matrices (see Definition 2.8 in $\S 3$ ). CO matrices were introduced as a more general class of matrices for which this eigenvalue relation holds. Analogous relations relating Jacobi eigenvalues to those of the corresponding SSOR iteration matrix have been obtained by Chong and Cai [3] for $\operatorname{GCO}(k, p-k)$ matrices and $\mathrm{Li}$ and Varga [13] for $\operatorname{GCO}(q, r)$ matrices. We note also that $\mathrm{CO}$ matrices possess property $A$ as defined by Young.

Young [24] conjectured, and Varga [21] proved, that orderings resulting in CO coefficient matrices were optimal in terms of rate of convergence for the SOR method with $\omega=1$, i.e., the Gauss-Seidel method. However, the usefulness of this theory is not limited solely to the use of SOR-type methods. For example, Harrar and Ortega [9] used the fact that a 2-cyclic matrix is $\mathrm{CO}$ and a result relating the eigenvalues of the corresponding SOR and SSOR iteration matrices, to derive an optimality result for the relaxation parameter $\omega$ in the context of the $m$-step SSOR PCG method. We note that the effect of consistent ordering, if any, on the rate of convergence of SSOR PCG methods is not known. For more details on the motivation for desiring that a matrix be CO, see Harrar [8] and, of course, Young [25].

In the sequel, we are concerned primarily with the property of being CO for block $p \times p$ matrices of the form (2). To this end we have a weaker version of consistent ordering.

Definition 2.3. Let the matrix $A$ be partitioned as in (2) and define a $p \times p$ matrix $Z=\left(z_{r s}\right)$ by

$$
z_{r s}= \begin{cases}0 & \text { if } A_{r s}=0 \\ 1 & \text { if } A_{r s} \neq 0\end{cases}
$$

The matrix $A$ is $\pi_{p^{-}}$consistently ordered $\left(\pi_{p^{-}} C O\right)$ if $Z$ is consistently ordered.

We note that, according to our notation, an $(n \times n)$-CO matrix is also $\pi_{n}$-CO.

Analogous to Definition 2.1 for a compatible ordering vector, we now introduce the concept of a $\pi_{p}$-compatible ordering vector for a block $p \times p$ matrix. This definition is intimated in Young [25]; we formalize it here. 
Definition 2.4. The vector $\gamma=\left(\gamma_{1}, \gamma_{2}, \ldots, \gamma_{p}\right)^{T}$, where the $\gamma_{i}$ are integers, is a $\pi_{p}$-compatible ordering vector for the block $p \times p$ matrix $A$ if, for $Z$ corresponding to $A$, as defined in (4), (3) holds.

We state without proof the following analog of Theorem 2.2.

THEOREM 2.5. A block $p \times p$ matrix $A$ is $\pi_{p}-C O$ if and only if there exists for $A$ a $\pi_{p}$-compatible ordering vector.

Obviously, a matrix of the form (2) can be $\pi$-CO and still not be CO. For example, a full $4 \times 4$ matrix partitioned as a block $2 \times 2$ matrix is $\pi_{2}-\mathrm{CO}$ but is not $\mathrm{CO}$, since we cannot construct a compatible ordering vector for it. This is because in such a matrix we have $a_{i j} \neq 0$ for all indices $i, j$, and the following observation holds.

OBSERVATION 2.6. It is impossible to construct a $\left(\pi_{p}\right)$-compatible ordering vector for a matrix of (block) order $p>2$, all of whose (block) elements are nonzero; that is, no (block) full matrix of (block) order $p>2$ is $\left(\pi_{p}\right)$-CO.

We also note the following.

OBSERVATION 2.7. All matrices of order greater than one are $\pi_{2}$-CO.

As shown in Theorems 2.9 and 2.10 below, one type of matrix of the form (2) that is both $\pi-\mathrm{CO}$ and $\mathrm{CO}$ is a T-matrix.

DEFINITION 2.8. A matrix with the block tridiagonal form

$$
T_{p}=\left[\begin{array}{ccccc}
D_{1} & H_{1} & & & \\
K_{1} & \cdot & \cdot & & \\
& \cdot & \cdot & \cdot & \\
& & \cdot & \cdot & H_{p-1} \\
& & & K_{p-1} & D_{p}
\end{array}\right],
$$

where the $D_{i}$ are square diagonal matrices, is a $T_{p}$-matrix.

It is easily verified that $\gamma=(1,2, \ldots, p)^{T}$ is a $\pi_{p}$-compatible ordering vector for a matrix of the form (5) where the $D_{i}$ need not be diagonal. Thus we have the following theorem.

THEOREM 2.9. A block $p \times p$ tridiagonal matrix is a $\pi_{p}-C O$ matrix.

(This is proved in a different manner by Hageman and Young [6].) Of course, an immediate corollary is that $T_{p}$-matrices are $\pi_{p}$-CO.

Now, although block tridiagonal matrices are not generally $\mathrm{CO}, T$-matrices are.

ThEOREM 2.10 (Young [25, Thm. 5.3.1]). A T-matrix is a CO matrix.

The property of being $\pi$-CO is important because it is often the case that, although a given matrix may not be $\mathrm{CO}$, it is $\pi$-CO for some partitioning $\pi$ of $A$ into blocks. And, in such circumstances, we may apply the results of the classical SOR theory to the partitioned matrix, i.e., we obtain an eigenvalue relation between the eigenvalues of the Jacobi and SOR iteration matrices, respectively, corresponding to the block partitioning of $A$. Of course, since the eigenvalues of the block Jacobi iteration matrix are a function of the matrix elements, it is generally nontrivial to compute them. But, for example, we can compute the optimal relaxation parameter $\omega_{\text {opt }}$ and the corresponding spectral radius $\rho_{\text {opt }}$ for line SOR methods (see $§ 5.1$ ).

3. The addition of block bidiagonal matrices $B_{r}$ to $T_{p}$. In this section we investigate what types of matrices can be added to block tridiagonal matrices (and hence also to $T$-matrices) so that the resulting matrix sum is still $\pi$-CO or even CO. This section is divided into three subsections. First, we examine the case in which the block tridiagonal matrix has no zero blocks on the first sub- or superdiagonal, that is, $H_{i} \neq 0$ or $K_{i} \neq 0$ for $i=1, \ldots, p-1$ in (5). Next, we consider the case in which the block $p \times p$ tridiagonal matrix has intermittent zero blocks on these diagonals, 
say $H_{k q}=0, K_{k q}=0$ for $k=1, \ldots, r$ where $r=p / q$ and $q$ is some integer that divides $p$ evenly; the generalization to the case for which these zero blocks are spaced nonuniformly should be obvious. Finally, we investigate the case in which the block tridiagonal matrix is a $T$-matrix.

Note throughout this paper that all results on $\pi$-consistent ordering for block matrices imply concomitant corollaries for the case of consistent ordering of nonblock matrices as a result of the correspondence inherent in Definition 2.3. That is, all results for block $p \times p$ matrices with blocks $A_{i j}$ in terms of $\pi$-consistent ordering imply exactly analogous results for $n \times n$ (where $n=p$ ) matrices with elements $a_{i j}$ in terms of consistent ordering.

3.1. $T_{p}$ has no zero blocks on the first sub- or superdiagonal. Here we consider the class of block $p \times p$ tridiagonal matrices $T_{p}$ which have no zero blocks on either the first subdiagonal or the first superdiagonal. We find that, for the matrix sum $T_{p}+A_{p}$ to be $\pi_{p}$-CO, $A_{p}$ must also be block tridiagonal. Although somewhat obvious, we state the result as a theorem in order to facilitate reference to it below. The proof illustrates the general method of proof used throughout.

THEOREM 3.1. Let $T_{p}$ be a block tridiagonal matrix of the form (5) where the $D_{i}$ are not necessarily diagonal, and suppose that $A_{p}$ has the block $p \times p$ form (2) where the blocks are partitioned commensurately with those of $T_{p}$. If $H_{i} \neq 0$ and $H_{i} \neq-A_{i, i+1}$, or $K_{i} \neq 0$ and $K_{i} \neq-A_{i+1, i}$, for $i=1, \ldots, p-1$, then the matrix $T_{p}+A_{p}$ is a $\pi_{p}-C O$ matrix if and only if $A_{i j}=0$ if $i>j+1$ or $j>i+1$, i.e., $A_{p}$ is block tridiagonal.

Proof. Clearly the sum of block tridiagonal matrices is also block tridiagonal, so that if $A_{p}$ is, then so is $T_{p}+A_{p}$. Therefore, $T_{p}+A_{p}$ is $\pi_{p}$-CO by Theorem 2.9.

Now, assume that $T_{p}+A_{p}$ is $\pi_{p} \mathrm{CO}$, and suppose that $A_{i j} \neq 0$ for some $i, j$ with, without loss of generality, $j>i+1$. Since $T_{p}+A_{p}$ is $\pi_{p}$-CO, we can, by Theorem 2.5 construct a $\pi_{p}$-compatible ordering vector $\gamma$ for $T_{p}+A_{p}$. Now, either $\left[T_{p}+A_{p}\right]_{i, i+1}=$ $H_{i}+A_{i, i+1} \neq 0$ or $\left[T_{p}+A_{p}\right]_{i+1, i}=K_{i}+A_{i+1, i} \neq 0$, for $i=1, \ldots, p-1$ since $H_{i} \neq-A_{i, i+1}$ or $K_{i} \neq-A_{i+1, i}$, respectively. Therefore, we must have $\gamma_{i+1}-\gamma_{i}=1$ for $i=1, \ldots, p-1$. Now, $\left[T_{p}+A_{p}\right]_{i j}=A_{i j} \neq 0$, so that we also require $\gamma_{j}-\gamma_{i}=1$. However,

$$
\gamma_{j}-\gamma_{i}=\left(\gamma_{j}-\gamma_{i+1}\right)+\left(\gamma_{i+1}-\gamma_{i}\right)=\left(\gamma_{j}-\gamma_{i+1}\right)+1>1
$$

since $j>i+1$, a contradiction. Therefore, we must have $A_{i j}=0$ for $j>i+1$. The case $A_{i j} \neq 0$ with $i>j+1$ is exactly analogous.

We note that, under the assumptions of Theorem 3.1, if $T_{p}$ and $A_{p}$ are $T$-matrices, then $T_{p}+A_{p}$ is $\mathrm{CO}$ by Theorem 2.10 since the sum of $T$-matrices is a $T$-matrix. However, the corresponding "only if" part of the theorem does not hold, in general, for $T$-matrices and consistent ordering. Although it is possible to add matrices other than $T$-matrices to a $T$-matrix to obtain a $C O$ matrix, the only type we can add without knowing anything about the internal structure of the off-diagonal blocks of $T$ and $A$ is a $T$-matrix.

The following is an immediate corollary of Theorem 3.1.

Corollary 3.2. Let the $n \times n$ matrix $A$ be such that all of the elements on the first sub-or superdiagonal are nonzero. Then $A$ is $C O$ if and only if $a_{i j}=0$ for $j>i+1$ and $i>j+1$.

3.2. $T_{p}$ has intermittent zero blocks on the first sub- and superdiagonal. We now consider block tridiagonal matrices that have intermittent zero blocks on the 
first off-diagonals. That is, we let the matrix $T$ have the block $r \times r$ diagonal form

$$
T=\operatorname{diag}\left(T_{11}, T_{22}, \ldots, T_{r r}\right),
$$

where the $q \times q$ diagonal blocks $T_{k k}$ are of the form

$$
T_{k k}=\left[\begin{array}{ccccc}
A_{l+1, l+1} & A_{l+1, l+2} & & & \\
A_{l+2, l+1} & \cdot & \cdot & & \\
& \cdot & \cdot & \cdot & A_{l+q-1, l+q} \\
& & \cdot & \cdot & A_{l+q, l+q-1} \\
& & & A_{l+q, l+q}
\end{array}\right]
$$

for $k=1, \ldots, r$, where $l=(k-1) q$ and $p=q r$. According to our notational convention, $T=T_{r}=T_{p}$, where $T_{r}$ is block $r \times r$ unidiagonal with $q \times q$ blocks and $T_{p}$ is block $p \times p$ tridiagonal with zero blocks every $q$ th entry on the first sub- and superdiagonals. Since $T_{p}$ is block tridiagonal, it is $\pi_{p}$-CO by Theorem 2.9 (it is also trivially $\pi_{r}$-CO). Of course, if the diagonal blocks $A_{l+i, l+i}, i=1, \ldots, q$ are square diagonal matrices, i.e., $A_{l+i, l+i}=D_{l+i}$ in (7), then $T_{p}$ given by (6), (7) is also a $T_{p}$-matrix and hence CO by Theorem 2.10 .

Now consider the class of block $r \times r$ bidiagonal matrices of the form

$$
B_{r}^{m}=\left[\begin{array}{ccccc}
0 & B_{1,2}^{m} & & & \\
B_{2,1}^{m} & \cdot & \cdot & & \\
& \cdot & \cdot & \cdot & \\
& & \cdot & \cdot & B_{r-1, r}^{m} \\
& & & B_{r, r-1}^{m} & 0
\end{array}\right]
$$

where the $q \times q$ nonzero blocks $B_{k, k+1}^{m}, k=1, \ldots, r-1$, may have only one nonzero block lower diagonal $\left(m=m_{L}\right)$

$$
B_{k, k+1}^{m_{L}}=L_{k, k+1}^{m_{L}}=\left[\begin{array}{llll}
A_{l+m_{L}, k q+1} & & & \\
& \cdot & & \\
& \cdot & \\
& & \cdot & \\
& & & A_{k q,(k+1) q-\left(m_{L}-1\right)}
\end{array}\right],
$$

where $m_{L}=2, \ldots, q$, or a nonzero block main diagonal $\left(m_{L}=1\right.$ in $\left.(9)\right)$, or one nonzero block upper diagonal $\left(m=m_{U}\right)$

$$
B_{k, k+1}^{m_{U}}=U_{k, k+1}^{m_{U}}=\left[\begin{array}{llll}
A_{l+1, k q+m_{U}} & & & \\
& \cdot & & \\
& \cdot & & \\
& & \cdot & A_{k q-\left(m_{U}-1\right),(k+1) q} \\
& & & \\
& & &
\end{array}\right],
$$

where $m_{U}=2, \ldots, q$. The blocks $B_{k+1, k}^{m}, k=1, \ldots, r-1$ have the same block structure as the blocks $\left(B_{k, k+1}^{m}\right)^{T}$. Note that $T_{p}$ is block tridiagonal of block order $p$ while $B_{r}^{m}$ is block bidiagonal of block order $r=p / q$; the block orders are different. 
We now have the following lemma.

LEMMA 3.3. Let $T_{p}$ be given by (6), (7) and $B_{r}^{m}$ be given by (8) with (9) or (10). Then the matrix $T_{p}+B_{r}^{m}$ is $\pi_{p}$-CO for all values of

$$
m=\left\{\begin{array}{l}
m_{L}=1, \ldots, q \\
m_{U}=2, \ldots, q
\end{array}\right.
$$

Proof. We show that the matrices $T_{p}+B_{r}^{m}$ are $\pi_{p}$-CO by showing the existence of $\pi_{p}$-compatible ordering vectors $\gamma$. We treat separately the cases in which the blocks $B_{k, k+1}^{m}$ have the form (9) or (10), i.e., $m=m_{L}$ or $m=m_{U}$, respectively. First, assume that $B_{k, k+1}^{m}=L_{k, k+1}^{m_{L}}$, so that they have the form (9). In this case a $\pi_{p}$-compatible ordering vector for $T_{p}+B_{r}^{m_{L}}$ is given by

$$
\begin{gathered}
\gamma^{L}=\left[1, \ldots, q, m_{L}+1, \ldots, m_{L}+q, 2 m_{L}+1, \ldots, 2 m_{L}+q, \ldots,\right. \\
\left.(r-1) m_{L}+1, \ldots,(r-1) m_{L}+q\right]^{T}
\end{gathered}
$$

or, in a somewhat more compact form notationally,

$$
\gamma^{L}=\left[\left((k-1) m_{L}+1,(k-1) m_{L}+2, \ldots,(k-1) m_{L}+q\right), k=1, \ldots, r\right]^{T} .
$$

Now, suppose that $m=m_{U}$, i.e., $B_{k, k+1}^{m}=U_{k, k+1}^{m_{U}}$ has the form (10). Then we obtain the $\pi_{p}$-compatible ordering vector

$$
\begin{gathered}
\gamma^{U}=\left[\left((k-1)\left(m_{U}-2\right)+1,(k-1)\left(m_{U}-2\right)+2, \ldots,\right.\right. \\
\left.\left.(k-1)\left(m_{U}-2\right)+q\right), k=1, \ldots, r\right]^{T}
\end{gathered}
$$

for $T_{p}+B_{r}^{m_{U}}$. We may verify that the vectors $\gamma^{L}$ and $\gamma^{U}$ given by (12) and (13), respectively, are $\pi_{p}$-compatible ordering vectors for the matrices $T_{p}+B_{r}^{m}$ where the blocks $B_{k, k+1}^{m}$ of $B_{r}^{m}$ have the form (9) and (10), respectively. Thus, by Theorem 2.5, $T_{p}+B_{r}^{m}$ is $\pi_{p}$-CO for all values of $m$ given by (11).

The regularity among the elements of the $\pi_{p}$-compatible ordering vectors for the matrices $T_{p}+B_{r}^{m}$ is quite striking. Note that the elements of these vectors corresponding to a given block $B_{k, k+1}^{m}$ are consecutive integers beginning with the $(k-1) q+1$ st element of the vector; this is true for $k=1, \ldots, r$. That is, we have

$$
\gamma_{(k-1) q+i}=\gamma_{(k-1) q+i-1}+1, \quad i=2, \ldots, q, \quad k=1, \ldots, r .
$$

Therefore, for a given $k$, the only element of $\gamma$ that depends on elements corresponding to another value of $k$ is the $(k-1) q+1$ st; the rest of the elements for that given $k$ can be obtained using (14). This suggests that it may be possible to construct $\pi_{p^{-}}$ compatible ordering vectors for matrices $T_{p}+B_{r}\left(T_{p}\right.$ given by (6), (7)) where the matrix $B_{r}$ now has the somewhat more general block $r \times r$ bidiagonal form

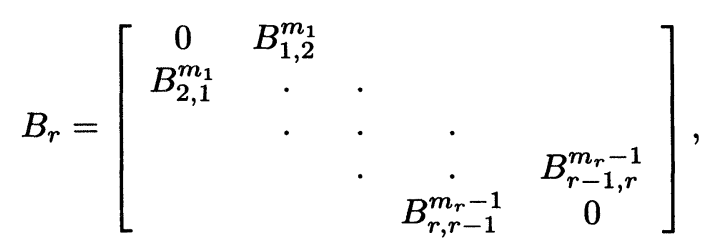

where each of the $q \times q$ nonzero blocks $B_{k, k+1}^{m_{k}}, i=1, \ldots, r-1$, has the form (9) or (10); that is, each $m_{k}$ can take on any value $m$ in (11). 
Lemma 3.4. Let $T_{p}$ be given by (6), (7) and let $B_{r}$ be given by (15) with each $B_{k, k+1}^{m_{k}}$ given by (9) or (10), and where each $m_{k}$ takes on a value of $m$ from (11). Then the matrix $T_{p}+B_{r}$ is $\pi_{p}$-CO for all choices of $B_{r}$, i.e., for all combinations of the $m_{k}$.

Proof. Using the $\pi_{p}$-compatible ordering vectors which we constructed in the proof of Lemma 3.3, we show how to construct a $\pi_{p}$-compatible ordering vector for $T_{p}+B_{r}$, given any $B_{r}$ of the form (15). Consider the block $B_{k, k+1}^{m_{k}}$. If this block has the form (9) so that $m_{k}=m_{L}$ for some $m_{L}$, then we notice from (12) that in going from $\gamma_{k q}^{L}$ to $\gamma_{k q+1}^{L}$, we need only add $m_{L}$ to $\gamma_{(k-1) q+1}^{L}$. If $B_{k, k+1}^{m_{k}}$ has the block strictly upper triangular form (10), we see that $\gamma_{k q+1}^{U}$ can be obtained by subtracting $m_{U}-2$ from $\gamma_{(k-1) q+1}^{U}$. In summary, then, we have

$$
\gamma_{k q+1}= \begin{cases}\gamma_{(k-1) q+1}+m_{k}, & \text { if } m_{k}=m_{L} \\ \gamma_{(k-1) q+1}-\left(m_{k}-2\right), & \text { if } m_{k}=m_{U}\end{cases}
$$

Of course, as noted above, the remaining elements are contiguous and would be calculated using (14). Now, the construction of a $\pi_{p}$-compatible ordering vector for $T_{p}+B_{r}$ proceeds as follows. We take as the first $q$ elements of the vector $\gamma$ the integers $1, \ldots, q$, i.e., $\gamma_{i}=i, i=1, \ldots, q$. Next, we consider the block $B_{1,2}^{m_{1}}$. If this block is block lower triangular, then we set $\gamma_{q+1}=\gamma_{1}+m_{1}=1+m_{1}, \gamma_{q+i}=\gamma_{q+i-1}+1, i=$ $2, \ldots, q$. However, if $B_{1,2}^{m_{1}}$ is block strictly upper triangular, we cannot simply use (16) to calculate $\gamma_{q+1}$ since for $m_{1}>2$ we would obtain a value for $\gamma_{q+1}$ which was nonpositive. To mitigate this problem, we would add $m_{1}-2$ to the first $q$ elements of $\gamma$, then calculate $\gamma_{q+1}$ using (16) and the next $q-1$ elements again using (14); we can do this because, clearly from Definition 2.1 , if $\gamma$ is a $\pi$-compatible ordering vector, then so is $\gamma+\delta$ where $\delta$ is any constant vector. With the blocks $B_{k, k+1}^{m_{k}}, k=2, \ldots, r-1$, we proceed in exactly the same fashion obtaining $\gamma_{k q+1}$ from $\gamma_{(k-1) q+1}$ using (16) with $m=m_{k}$ and then using (14) to calculate the next $q-1$ elements of $\gamma$. If $B_{k, k+1}^{m_{k}}$ has the form (10), we first check if $\gamma_{k q+1}=\gamma_{(k-1) q+1}-\left(m_{k}-2\right)>0$; if not, then we first add $m_{k}-2$ to the thus far computed $k q$ elements of $\gamma$. When we have proceeded through all of the blocks $B_{k, k+1}^{m_{k}}$, we have constructed a $\pi_{p}$-compatible ordering vector for $T_{p}+B_{r}$, thus, by Theorem $2.5, T_{p}+B_{r}$ is $\pi_{p}$-CO.

We now show that, if $B_{r}$ is of the form (15), then $T_{p}+B_{r}$ cannot be $\pi_{p}$-CO unless each of the blocks $B_{k, k+1}^{m_{k}}, k=1, \ldots, r-1$, has one of the unidiagonal forms (9), (10).

TheOREM 3.5. Let $T_{p}$ be given by (6), (7) and let $B_{r}$ be given by (15). Then the matrix sum $T_{p}+B_{r}$ is $\pi_{p}$-CO if and only if each $B_{k, k+1}^{m_{k}}, k=1, \ldots, r-1$, is given by (9) or (10), where each $m_{k}$ takes on a value of $m$ from (11).

Proof. If each $B_{k, k+1}^{m_{k}}$ is given by (9) or (10) with $m_{k}$ taking on a value of $m$ from (11), then $T_{p}+B_{r}$ is $\pi_{p}$-CO by Lemma 3.4 .

We now show that if any of the blocks $B_{k, k+1}^{m_{k}}$ has a form different from (9) and (10), then it is impossible to construct a $\pi_{p}$-compatible ordering vector for $T_{p}+B_{r}$; thus the matrix sum $T_{p}+B_{r}$ is not $\pi_{p}$-CO by Theorem 2.5. Consider the block $B_{k, k+1}^{m_{k}}$ where $k$ is now fixed and is chosen from the range of values $k=1, \ldots, r-1$. We assert that given one nonzero block $A_{l+i, k q+j}$ (recall that $l=(k-1) q$ ), where $i, j \in\{1, \ldots, q\}$, in $B_{k, k+1}^{m_{k}}$, the only other blocks of $B_{k, k+1}^{m_{k}}$ which can be nonzero are those lying along the diagonal of which $A_{l+i, k q+j}$ is a member.

We treat two cases: In Case $1(i \geq j), A_{l+i, k q+j}$ is in the lower triangular portion of $B_{k, k+1}^{m_{k}}$ or on the main diagonal of $B_{k, k+1}^{m_{k}}$. In Case $2(i<j), A_{l+i, k q+j}$ is in the upper triangular portion of $B_{k, k+1}^{m_{k}}$. 
Case $1(i \geq j)$. From (9) we see that $B_{k, k+1}^{m_{k}}=B_{k, k+1}^{m_{L}}$ where $m_{L}=i-j+1$ and $A_{l+i, k q+j} \neq 0$ for some $i, j$ where $m_{L} \leq i \leq q$ and $1 \leq j \leq q-\left(m_{L}-1\right)$. The parameter $m_{L}$ uniquely determines the diagonal of which $A_{l+i, k q+j}$ is a member, and it can be seen that all elements of this diagonal are of the form $A_{l+i, k q+j}$ where the integer pair $(i, j)$ is a member of the set

$$
\Lambda_{m_{L}}=\left\{(i, j) \mid m_{L} \leq i \leq q, 1 \leq j \leq q-\left(m_{L}-1\right) \text { and } i-j=m_{L}-1\right\} .
$$

Assume now that, for some $\xi, \eta \in\{1, \ldots, q\}, A_{l+\xi, k q+\eta} \neq 0$ and $(\xi, \eta) \notin \Lambda_{m_{L}}$. Then, in order that a $\pi_{p}$-compatible ordering vector exist, the requirement (3) becomes

$$
\gamma_{l+\xi}-\gamma_{k q+\eta}=\left\{\begin{aligned}
1 & \text { if } l+\xi>k q+\eta \\
-1 & \text { if } l+\xi<k q+\eta
\end{aligned}\right.
$$

However, since $\xi, \eta \in\{1, \ldots, q\}$, we can never have $l+\xi>k q+\eta$. Noting that $k q-l=k q-(k-1) q=q$ so that $l+\xi<k q+\eta$ is always satisfied, we thus require

$$
\gamma_{l+\xi}-\gamma_{k q+\eta}=-1
$$

Now, since the elements of $\gamma$ are consecutive for indices from $l+1$ to $k q$,

$$
\begin{aligned}
\gamma_{l+\xi} & =\left(\gamma_{l+\xi}-\gamma_{l+\xi-1}\right)+\left(\gamma_{l+\xi-1}-\gamma_{l+\xi-2}\right)+\cdots+\left(\gamma_{l+2}-\gamma_{l+1}\right)+\gamma_{l+1} \\
& =(\xi-1)+\gamma_{l+1} .
\end{aligned}
$$

Similarly, the elements of $\gamma$ are consecutive for indices from $k q+1$ to $(k+1) q$ so that

$$
\begin{aligned}
\gamma_{k q+\eta} & =\left(\gamma_{k q+\eta}-\gamma_{k q+\eta-1}\right)+\left(\gamma_{k q+\eta-1}-\gamma_{k q+\eta-2}\right)+\cdots+\left(\gamma_{k q+2}-\gamma_{k q+1}\right)+\gamma_{k q+1} \\
& =(\eta-1)+\gamma_{k q+1}
\end{aligned}
$$

Subtracting (20) from (19) and using the first line of (16), we have

$$
\gamma_{l+\xi}-\gamma_{k q+\eta}=(\xi-\eta)+\gamma_{l+1}-\gamma_{k q+1}=(\xi-\eta)-m_{L}
$$

Substituting into (18) this gives

$$
\xi-\eta=m_{L}-1 .
$$

But then, from the definition of $\Lambda_{m_{L}}$, we would have $(\xi, \eta) \in \Lambda_{m_{L}}$, a contradiction. Therefore, we must have $A_{l+\xi, k q+\eta}=0$ for $(\xi, \eta)$ not in $\Lambda_{m_{L}}$.

Case $2(i<j)$. In this case we assume that the block $B_{k, k+1}^{m_{k}}$ contains a nonzero block $A_{l+i, k q+j}$, where now $1 \leq i \leq q-\left(m_{U}-1\right)$ and $m_{U} \leq j \leq q$. Thus $B_{k, k+1}^{m_{k}}$ has the form $B_{k, k+1}^{m_{U}}$ where $m_{U}=j-i+1$. From (10) we see that for any element $A_{l+i, k q+j}$ of the diagonal determined by $m_{U}$ the integer pair $(i, j)$ is a member of the set

$$
\Upsilon_{m_{U}}=\left\{(i, j) \mid 1 \leq i \leq q-\left(m_{U}-1\right), m_{U} \leq j \leq q \text { and } j-i=m_{U}-1\right\} .
$$

Now, assume that $A_{l+\xi, k q+\eta} \neq 0$ for some $\xi, \eta \in\{1, \ldots, q\}$ such that $(\xi, \eta) \notin \Upsilon_{m_{U}}$. Then, analogous to Case 1 , in order that a $\pi_{p}$-compatible ordering vector exist for $T_{p}+B_{r}$, we again obtain the requirement (18). Using (19) and (20) and the third line of (16), from which $\gamma_{l+1}-\gamma_{k q+1}=m_{U}-2$, (21) becomes

$$
\gamma_{l+\xi}-\gamma_{k q+\eta}=(\xi-\eta)+m_{U}-2
$$


Substituting this into (18) we get

$$
\xi-\eta=1-m_{U}
$$

so that $(\xi, \eta)$ is in $\Upsilon_{m_{U}}$, a contradiction. Thus $A_{l+\xi, k q+\eta}=0$ for $(\xi, \eta)$ not in $\Upsilon_{m_{U}}$. Therefore, we conclude that, in order that a $\pi_{p}$-compatible ordering vector exist for $T_{p}+B_{r}$ so that $T_{p}+B_{r}$ is $\pi_{p}$-CO, the nonzero blocks $B_{k, k+1}^{m_{k}}$ of $B_{r}$ must, for each $k$, have one of the unidiagonal forms (9), (10), and the proof is complete.

3.3. $T_{p}$ is a $T_{p}$-matrix. Now, we consider the case in which $T_{p}$ is a $T_{p}$-matrix with intermittent zeros on the first sub- and superdiagonal. Of course, in this case, a matrix of the form $T_{p}+B_{r}$ is still $\pi_{p}$-CO by Theorem 3.5, but it turns out that such a matrix is also CO.

THEOREM 3.6. Suppose that the diagonal blocks $A_{l+i, l+i}$ of $(7)$ are square diagonal matrices, and let $B_{r}$ be a block bidiagonal matrix of the form (15). Then any matrix of the form $T_{p}+B_{r}$ where $T_{p}$ is given by (6), (7) is $C O$ if each of the blocks $B_{k, k+1}^{m}$ of $B_{r}$ is given by one of the unidiagonal forms (9), (10).

Proof. If each $B_{k, k+1}^{m_{k}}$ is given by (9) or (10) with $m_{k}$ taking on a value of $m$ from (11), then we show that we can easily construct a compatible ordering vector for $T_{p}+B_{r}$ using our previous results. Let $s$ denote the order of the diagonal blocks $A_{l+i, l+i}$. (The case in which these blocks each have different order, say $s_{l+i}$, is no more difficult to prove; however, the subscripting becomes overly cumbersome.) We assert that, in order to construct a compatible ordering vector $\gamma$ for $T_{p}+B_{r}$, we must only take the $\pi_{p}$-compatible ordering vector (which we now denote $\gamma^{\pi}$ ) for $T_{p}+B_{r}$, constructed as in the proof of Lemma 3.4, and repeat each element $s$ times. That is, with $l=(k-1) q$, we set

$$
\gamma_{(l+i-1) s+\hat{\imath}}=\gamma_{l+i}^{\pi}, \quad \hat{\imath}=1, \ldots, s ; \quad i=1, \ldots, q ; \quad k=1, \ldots, r .
$$

In order to verify that $\gamma$ constructed in this manner is a compatible ordering vector for $T_{p}+B_{r}$, we must show that (3) holds for all nonzero elements $a_{g h}$ of $T_{p}+B_{r}$; note that $g, h \in\{1, \ldots, n\}$ where $n=q r s$. There are two situations that we must treat: $a_{g h} \neq 0$ represents an element of one of the off-diagonal blocks $A_{l+i, l+i+1}$ of (7) and $a_{g h} \neq 0$ represents an element of some $A_{l+i, k q+j}$ of $B_{k, k+1}^{m_{k}}$. Consider the case in which $a_{g h} \neq 0$ is an element of one of the off-diagonal blocks of (7), $A_{l+i, l+i+1}$. Then we have

$$
g=(l+i-1) s+\hat{\imath}, \quad h=(l+i) s+\hat{\jmath},
$$

where $\hat{\imath}, \hat{\jmath} \in\{1, \ldots, s\}$. So, requirement (3) becomes

$$
\gamma_{g}-\gamma_{h}=\gamma_{(l+i-1) s+\hat{\imath}}-\gamma_{(l+i) s+\hat{\jmath}}=\left\{\begin{aligned}
1 & \text { if }(l+i-1) s+\hat{\imath}>(l+i) s+\hat{\jmath} \\
-1 & \text { if }(l+i-1) s+\hat{\imath}<(l+i) s+\hat{\jmath} .
\end{aligned}\right.
$$

Since $\hat{\imath}, \hat{\jmath} \in\{1, \ldots, s\}$, we can never have $(l+i-1) s+\hat{\imath}>(l+i) s+\hat{\jmath}$, so we require that

$$
\gamma_{(l+i-1) s+\hat{\imath}}-\gamma_{(l+i) s+\hat{\jmath}}=-1
$$

By (23), this is equivalent to requiring that

$$
\gamma_{l+i}^{\pi}-\gamma_{l+i+1}^{\pi}=-1
$$


which is true in our construction of $\gamma^{\pi}$ by (14).

Now, we consider the case in which $a_{g h} \neq 0$ is in a block $A_{l+i, k q+j}$ of $B_{k, k+1}^{m_{k}}$. Then we have

$$
g=(l+i-1) s+\hat{\imath}, \quad h=(k q+j-1) s+\hat{\jmath}
$$

for some $\hat{\imath}, \hat{\jmath} \in\{1, \ldots, s\}$. Proceeding as before, we obtain the requirement

$$
\gamma_{(l+i-1) s+\hat{\imath}}-\gamma_{(k q+j-1) s+\hat{\jmath}}=-1
$$

By (23), we thus require that

$$
\gamma_{l+i}^{\pi}-\gamma_{k q+j}^{\pi}=-1
$$

This is the requirement (18) with $\xi=i, \eta=j$, which we found to hold if and only if $(\xi, \eta)=(i, j)$ is in $\Lambda_{m_{L}}$ or $\Upsilon_{m_{U}}$, depending on whether $m_{k}=m_{L}$ or $m_{k}=m_{U}$, that is, if and only if $A_{l+i, k q+j}$ lies along the diagonal determined by $m_{k}$. This is true by assumption; thus (3) holds for $\gamma_{g}$ and $\gamma_{h}$ corresponding to $a_{g h}$.

Hence, for any nonzero element of either of the two "types" of nonzero blocks $\left(A_{l+i, l+i+1}\right.$ and $\left.A_{l+i, k q+j}\right)$ of $T_{p}+B_{r}$, the corresponding elements of $\gamma$ given by (23) satisfy the requirements set forth in Definition 2.1 of a compatible ordering vector. Therefore, using (23), where the elements $\gamma_{k q+i}^{\pi}$ are found as in the proof of Lemma 3.4, we can construct a compatible ordering vector for $T_{p}+B_{r}$, so it is a $\mathrm{CO}$ matrix by Theorem 2.2.

As was the case with Theorem 3.1 in $\S 3.1$, we cannot strengthen the above result to be an "if and only if" statement. Although the blocks $B_{k, k+1}^{m_{k}}$ of $B_{r}$ do not necessarily have to have one of the block unidiagonal forms $(9),(10)$ in order that the matrix sum $T_{p}+B_{r}$ (with $T_{p}$ a $T_{p}$-matrix) be CO, we can only use the method of constructing a compatible ordering vector given in the proof of Theorem 3.6 if the $B_{k, k+1}^{m_{k}}$ do have one of these unidiagonal forms. Otherwise, we need to know something about the internal structure of the $A_{l+i, l+i+1}$ of (7) and the $A_{l+i, k q+j}$ of $B_{k, k+1}^{m_{k}}$.

The method of proving Theorem 3.6 can be extended in a straightforward manner to prove the following stronger and very useful result.

TheOREM 3.7. Let the block $p \times p$ matrix $A$ be given by (2), and suppose that the diagonal blocks $A_{i i}, i=1, \ldots, p$ are diagonal matrices. If $A$ is $\pi_{p}-C O$, then $A$ is $C O$.

A proof of Theorem 3.7 would be similar to the following. If $A$ is $\pi_{p}$-CO, then by Theorem 2.5 there exists for $A$ a $\pi_{p}$-compatible ordering vector, say $\gamma^{\pi_{p}}$. Let $s_{i}$ denote the order of the diagonal block $A_{i i}$, for $i=1, \ldots, p$. Now construct a vector $\gamma$ by repeating each element $\gamma_{i}^{\pi_{p}}$ of $\gamma^{\pi_{p}} s_{i}$ times. Then, using the method of proof used for Theorem 3.6, we would show that the vector $\gamma$, consisting of $\sum_{i=1}^{p} s_{i}$ elements, is a compatible ordering vector for $A$. Thus $A$ is $\mathrm{CO}$ by Theorem 2.2 . We note that Young intimated this result by stating: If $A_{p}$ is $\pi_{p}$-CO, then $C_{p}=D_{p}-A_{p}$ is $\mathrm{CO}$, where the blocks $D_{i j}$ of $D_{p}$ are given by $D_{i i}=A_{i i}$ and $D_{i j}=0, i \neq j$ (Young [25, Thm. 14.3.2]).

Often, when using a multicoloring scheme, we obtain a matrix with diagonal blocks which are, in turn, diagonal. In this case, Theorem 3.7 provides an efficient way of showing whether or not that matrix is $\mathrm{CO}$ by simply finding a $\pi_{p}$-compatible ordering vector for it rather than a compatible ordering vector. In general, this should represent a substantial simplification.

We note that the following corollary of Theorem 3.7 may also be useful.

Corollary 3.8. Let the block $p \times p$ matrix $A$ have diagonal blocks $A_{i i}, i=$ $1, \ldots, p$, which are block diagonal of block order $s$. Assume that $A$ can also be partitioned as a block $t \times t$ matrix with $t=p s$. If $A$ is $\pi_{p}-C O$, then $A$ is $\pi_{t}-C O$. 
4. The addition of more general block matrices $M_{r}$ to $T_{p}$. The $\pi_{p}$-compatible ordering vectors constructed in the manner prescribed in $\S 3.2$ allow for even more nonzero blocks in the matrix sum; these nonzero blocks must again take one of the unidiagonal forms (9), (10). We consider the addition of a more general class of block matrices $M_{r}$ to block tridiagonal matrices $T_{p}$ where the $M_{r}$ have more than just two nonzero block diagonals. We consider matrices $M_{r}$ of the form

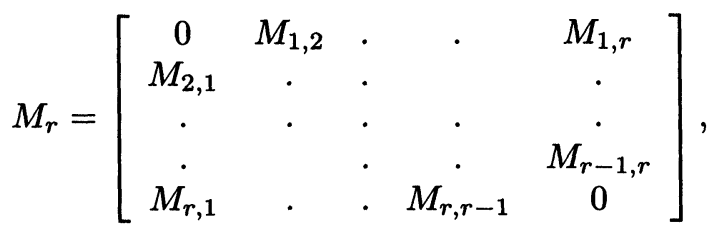

where we again assume that this matrix is symmetrically structured. That is, the nonzero block structure of a block $M_{i j}$ is the same as that of $M_{j i}^{T}$. In the language of previous sections, these matrices would be referred to as block "( $2 r-2)$-diagonal" matrices.

We denote the off-diagonal blocks of the matrix $M_{r}$ of (26) by $M_{k, k+i}$, where $k=1, \ldots, r$ and $i=1, \ldots, r-k$; associated with each of these blocks will be a value $m_{k, k+i}$, selected from (11), which will determine the unidiagonal structure. Thus $i$ serves as an index for the superdiagonal (and, by the symmetrical structure assumption, the associated subdiagonal) under consideration; the case $i=1$ was the subject of $\S 3$.

We begin with the case $i=2$. There are several possible situations. The allowable value of $m_{k, k+2}$ depends on the values of $m_{k, k+1}$ and $m_{k+1, k+2}$; that is, $m_{k, k+2}$ depends on the values of $m$ in the block to the left and in the block below the $k, k+2$ nd block. There are four possibilities, depending on whether these values of $m$ are of the form $m_{L}$ or $m_{U}$; the corresponding allowable values of $m_{k, k+2}$ for $\pi_{p^{-}}$ consistent ordering are given below. (In (27), (28) by $M_{k, k+2}^{m_{k, k+2}}=0$, we mean that $M_{k, k+2}^{m_{k, k+2}}$ has no nonzero elements.)

(i) $m_{k, k+1}=m_{L}=1, \ldots, q$ and $m_{k+1, k+2}=m_{L}=1, \ldots, q$,

$$
\begin{aligned}
& m_{k, k+1}+m_{k+1, k+2} \leq q \quad \Longrightarrow \quad m_{k, k+2}=m_{L}=m_{k, k+1}+m_{k+1, k+2} \\
& m_{k, k+1}+m_{k+1, k+2}>q \quad \Longrightarrow \quad M_{k, k+2}^{m}=0 .
\end{aligned}
$$

(ii) $m_{k, k+1}=m_{U}=2, \ldots, q$ and $m_{k+1, k+2}=m_{U}=2, \ldots, q$,

$$
\begin{aligned}
& m_{k, k+1}+m_{k+1, k+2}-2 \leq q \Longrightarrow m_{k, k+2}=m_{U}=m_{k, k+1}+m_{k+1, k+2}-2 \\
& m_{k, k+1}+m_{k+1, k+2}-2>q \Longrightarrow M_{k, k+2}^{m}=0 .
\end{aligned}
$$

(iii) $m_{k, k+1}=m_{L}=1, \ldots, q$ and $m_{k+1, k+2}=m_{U}=2, \ldots, q$,

$$
\begin{aligned}
& m_{k, k+1}>m_{k+1, k+2}-2 \quad \Longrightarrow \quad m_{k, k+2}=m_{L}=m_{k, k+1}-m_{k+1, k+2}+2 \\
& m_{k, k+1} \leq m_{k+1, k+2}-2 \quad \Longrightarrow \quad m_{k, k+2}=m_{U}=m_{k+1, k+2}-m_{k, k+1} .
\end{aligned}
$$

(iv) $m_{k, k+1}=m_{U}=2, \ldots, q$ and $m_{k+1, k+2}=m_{L}=1, \ldots, q$,

$$
\begin{aligned}
& m_{k+1, k+2} \leq m_{k, k+1}-2 \quad \Longrightarrow \quad m_{k, k+2}=m_{U}=m_{k, k+1}-m_{k+1, k+2} \\
& m_{k+1, k+2}>m_{k, k+1}-2 \quad \Longrightarrow \quad m_{k, k+2}=m_{L}=m_{k+1, k+2}-m_{k, k+1}+2 .
\end{aligned}
$$

For values of $i$ greater than 2, we use the values of any pair of $m$ 's, $m_{k, k+t}$ and $m_{k+t, k+i}$, where $0<t<i$. For instance, to obtain the allowable value of, 
say $m_{k, k+4}$, we could use any of the pairs $m_{k, k+1}, m_{k+1, k+4}$ or $m_{k, k+2}, m_{k+2, k+4}$ or $m_{k, k+3}, m_{k+3, k+4}$. The formulas to be used to obtain the allowable value of $m_{k, k+i}$ are exactly (27)-(30), except that we replace $k+1$ by $k+t$ and $k+2$ by $k+i$. Note, however, that when a block was set to zero as in the second lines of (i) and (ii) above, we do not assume a value of 0 for $m$ in that block; rather, $m$ carries the value indicated in the corresponding first line, although greater than $q$.

Proceeding in this manner, it is possible to check whether a block matrix is $\pi_{p^{-}}$ CO. If all of the diagonal blocks $A_{l+i, l+i}$ are diagonal matrices, then, by Theorem 3.7, we can check whether the matrix is $\mathrm{CO}$.

5. Application of the theory to some multicolor orderings. In this section we apply some of the results of $\S 3$ to some well-known and not so well-known orderings that appear in the literature. Although convergence properties for these orderings are beyond the scope of this paper, they can generally be found in the given references. In order to concretize some of what follows, we apply the discussion to the solution of the discretized analog of the two-dimensional Laplace problem

$$
\begin{aligned}
\nabla^{2} u=0 & \text { in } \quad \Omega=[0,1] \times[0,1] \\
u=0 & \text { on } \partial \Omega .
\end{aligned}
$$

In the discretization we assume that there are an even number $N$ of grid points in each direction.

5.1. Line and zebra orderings. One frequently used class of orderings is the class of line orderings. These are multicoloring schemes in which all of the points on a given line of the grid, or group of lines, has the same color. Thus, for example, for a two-dimensional problem on an $N \times N$ grid, a one-line ordering results in $N$ colors, while a $k$-line ordering gives $N / k$ colors (we generally choose $k$ so that it divides $N$ evenly).

Ordering the lines, or groups of lines, in the natural ordering from bottom to top, the coefficient matrix under the usual five-point finite difference discretization, as with a natural ordering of the grid points, has the form

$$
A=\operatorname{tridiag}(-I, T,-I), \quad T=\operatorname{tridiag}(-1,4-1) .
$$

(Notationally, by tridiag $(A, B, C)$, we mean the block tridiagonal matrix with matrices $B$ along the main diagonal, and matrices $A, C$ along the first sub- and superdiagonals, respectively.) Here $T$ is $N \times N$, and $I$ is the identity matrix of order $N$. For $k=1$, we have a block $N \times N$ structure. For general $k, A$ would be partitioned as a block $\frac{N}{k} \times \frac{N}{k}$ matrix of $k N \times k N$ blocks. In each case, the matrix is block tridiagonal of block order $\frac{N}{k}$ and hence is $\pi_{N / k}$-CO by Theorem 2.9 .

Next, consider a zebra ordering. We color all of the odd-numbered rows of the grid, say, black, and all of the even-numbered rows white. Within each color we then number the grid points in the natural ordering. Using a five-point stencil in the discretization of $(31)$, the coefficient matrix would have the red/black (block $2 \times 2$ ) form

$$
A=\left[\begin{array}{cc}
D_{1} & C \\
C^{T} & D_{2}
\end{array}\right], \quad D_{1}=D_{2}=\operatorname{diag}(T), \quad C=\operatorname{tridiag}(-I,-I, 0),
$$

and $T$ is given in (32). Here $\operatorname{diag}(T)$ is a block diagonal matrix with diagonal blocks T. $D_{i}, C$ are block $\frac{N}{2} \times \frac{N}{2}$ while $T, I$ are $N \times N$. Since $A$ is $\pi_{2}$-CO with $D_{1}, D_{2}$ block diagonal, Corollary 3.8 indicates that the coefficient matrix for a zebra ordering is also $\pi_{N}$-CO. 
5.2. Many-color red/black orderings. The many-color red/black orderings of Harrar and Ortega [10] are of two general types: row-wise red/black orderings and planar red/black orderings. The 1-row and $2 k$-row red/black orderings involve imposing a red/black ordering on every row or $2 k$ rows (lines) of the grid where $k=1,2, \ldots, N / 2$ and $N$ is the number of rows. For three-dimensional problems, we can also consider 1-plane and $2 k$-plane red/black orderings where a red/black ordering is imposed on every plane or $2 k$ planes of the grid, respectively. In either case, the red and black unknowns of a given color are numbered in a natural ordering.

In general, for a $2 k$-row red/black ordering the linear system is of the form

$$
\left[\begin{array}{ccccccc}
D_{1} & A_{12} & 0 & A_{14} & . & . & \cdot \\
A_{12}^{T} & D_{2} & A_{23} & 0 & \cdot & \cdot & . \\
0 & A_{23}^{T} & D_{3} & A_{34} & 0 & A_{36} \\
A_{14}^{T} & 0 & A_{34}^{T} & D_{4} & A_{45} & 0 \\
\cdot & \cdot & 0 & A_{45}^{T} & \cdot & & \\
\cdot & \cdot & A_{36}^{T} & & & \cdot & \\
\cdot & \cdot & & & & & .
\end{array}\right]\left[\begin{array}{c}
u_{R 1} \\
u_{B 1} \\
u_{R 2} \\
u_{B 2} \\
\cdot \\
\cdot \\
\cdot
\end{array}\right]=f .
$$

Here the $D_{i}$ are diagonal matrices, $u_{R i}$ is the vector of unknowns associated with the $R i$ grid points, and similarly for $u_{B i}$. For an $N \times N$ grid the coefficient matrix is block $\frac{N}{k} \times \frac{N}{k}$ with $k N \times k N$ blocks. For three-dimensional problems on an $N \times N \times N$ grid, a $2 k$-plane red/black ordering again yields a system of the form (34), where the coefficient matrix is block $\frac{N}{k} \times \frac{N}{k}$, except that now the blocks are $k N^{2} \times k N^{2}$ and consist of more nonzero diagonals.

The coefficient matrix of the system (34) is a matrix of the form $T_{p}+A_{p}$ where $p=N / k ; T_{p}$ is a $T_{p}$-matrix (a $T_{N / k}$-matrix), and $A_{p}$ is a block $\frac{N}{k} \times \frac{N}{k}$ matrix which has all zero blocks except for $A_{i, i+3}$ (and $A_{i+3, i}=A_{i, i+3}^{T}$ ) where $i=1,3, \ldots, N-3$. All of the blocks on the first sub- and superdiagonals of $T_{p}$ are nonzero. Thus, by Theorem 3.1, $T_{p}+A_{p}$ is not $\pi_{p}$-CO. In order to determine whether or not the coefficient matrix of (34) is $\mathrm{CO}$, we would need to investigate the internal structure of its offdiagonal blocks.

Now consider a 1-row red/black ordering. The system of equations corresponding to a five-point finite difference discretization of (31) would have the block $2 N \times 2 N$ form

$$
\left[\begin{array}{ccccccc}
D_{1} & A_{12} & A_{13} & 0 & \cdot & \cdot & \cdot \\
A_{12}^{T} & D_{2} & 0 & A_{24} & . & . & \cdot \\
A_{13}^{T} & 0 & D_{3} & A_{34} & A_{35} & & \\
0 & A_{24}^{T} & A_{34}^{T} & D_{4} & 0 & & \\
\cdot & \cdot & A_{35}^{T} & & \cdot & \\
\cdot & \cdot & & & & . & \\
\cdot & \cdot & & & & & .
\end{array}\right]\left[\begin{array}{c}
u_{R 1} \\
u_{B 1} \\
u_{R 2} \\
u_{B 2} \\
\cdot \\
\cdot \\
\cdot
\end{array}\right]=f,
$$

where each block is $\frac{N}{2} \times \frac{N}{2}$. This is also the form of the system of equations for the three-dimensional analog of (31) in the case of a 1-plane red/black ordering except that each block would be $N^{2} / 2 \times N^{2} / 2$ and the $A_{i, i+1}, i=1, \ldots, N-1$ would have more nonzero diagonals.

Using the nomenclature of $\S 3$, the 1-row (1-plane) red/black coefficient matrix of (35) is a matrix of the form $T_{p}+B_{r}$, where $p=2 N, r=N\left(p=2 N^{2}, r=N^{2}\right) . T_{p}$ is block tridiagonal with intermittent zero blocks every second block on the first suband superdiagonals. $B_{r}$ is block bidiagonal where each block $B_{k, k+1}^{m}$ is block $2 \times 2$ 
with $m=m_{L}=1$. This matrix is thus $\pi_{p}$-CO by Lemma 3.4. In fact, from the proof of Lemma 3.4, we see that a $\pi_{p}$-compatible ordering vector for this matrix is given by (12) with $q=2$, that is, $\gamma^{L}=(1,2,2,3, \ldots, 2 N, 2 N+1)^{T}$. (For the three-dimensional problem with a 1-plane red/black ordering, replace $N$ by $N^{2}$.) Since the diagonal blocks of $T_{p}$ are diagonal, Theorem 3.6 indicates that a compatible ordering vector for $T_{p}+B_{r}$ is given by

$$
\gamma^{L}=\left[(1)_{N / 2},(2)_{N / 2},(2)_{N / 2},(3)_{N / 2}, \ldots,(2 N)_{N / 2},(2 N+1)_{N / 2}\right]^{T}
$$

where $(i)_{s}$ denotes the $s$-long vector, all of whose elements are $i$. Thus we know that the matrix of (35) is $\mathrm{CO}$ without knowing anything about the internal structure of the off-diagonal blocks.

Returning to the $2 k$-row red/black orderings, suppose that we reorder the unknowns as

$$
u=\left(u_{R 1}, u_{B 1}, u_{B 2}, u_{R 2}, u_{R 3}, u_{B 3}, \ldots\right)^{T} .
$$

Then, the coefficient matrix has the same block form as the 1-row red/black matrix of (35), except that it is block $\frac{N}{k} \times \frac{N}{k}$ with $k N \times k N$ blocks. Thus we again obtain a $\pi_{p}$-CO matrix which is, in fact, also CO. Harrar and Ortega [10] discussed this reordering as a way to reduce the bandwidth of the coefficient matrix, but made no mention of $\mathrm{CO}$ (or $\pi$-CO) matrices.

5.3. Other orderings. In this section, we discuss some other orderings that have been proposed in the literature. The primary motivation for many of these orderings is the need for more than two colors to decouple the unknowns under a ninepoint grid stencil (for a two-dimensional problem); in this case it is well known that at least four colors are necessary. Adams and Jordan [1] identified 72 distinct four-color orderings which could be used to bring about this local decoupling of unknowns. All 72 of these four-color orderings lead to matrices which are neither CO nor $\pi_{4}$-CO.

Adams and Jordan [1] define a multicolor, or c-color, matrix to be a block matrix of the form (2) with $p=c$ and where the diagonal blocks $A_{i i}$ are diagonal matrices. They also define a multicolor T-matrix as a block tridiagonal matrix of the form

$$
T_{M}=\operatorname{tridiag}\left(U_{i-1}, M_{i}, L_{i}\right), \quad i=1, \ldots, s
$$

(where, of course, $U_{0}$ and $L_{s}$ do not appear in the first and last rows, respectively). In (37) the $M_{i}$ are multicolor matrices, the $L_{i}$ are block strictly lower triangular, and the $U_{i}$ have the transposed structure of $L_{i}$, respectively. Now, by Theorem 3.7, if a multicolor matrix as defined above is $\pi_{c}$-CO, it is CO. However, by Theorem 3.1, if $A_{i, i+1} \neq 0, i=1, \ldots, c-1$, then a multicolor matrix is not $\pi_{c}$-CO if any of the other $A_{i j}$ are nonzero. If some of the $A_{i, i+1}$ do consist solely of zero entries, then Theorem 3.5 and the theory of $\S 4$ may allow one to determine whether or not the multicolor matrix is $\pi_{c}$-CO. However, $T_{M}$ given by (37) is always $\pi_{s}$-CO by Theorem 2.9, and the main result of Adams and Jordan [1] is that $T_{M}$ and its associated multicolor matrix have corresponding SOR iteration matrices with the same eigenvalues. Thus, if the coloring is such that the multicolor matrix has an associated multicolor $T$-matrix $T_{M}$, we can apply the classical SOR theory to the $\pi_{s}$-CO matrix $T_{M}$ to gain information about the eigenvalues of the SOR iteration matrix associated with the multicolor matrix which may be neither $\pi$-CO nor CO.

Kuo and Levy [12] also consider four-color orderings for a nine-point discretization of a two-dimensional Poisson equation. Rather than analyzing the Jacobi iteration 
matrix in the space domain, they consider a simpler, yet equivalent, four-color iteration matrix in the frequency domain. This matrix is not $\pi_{4}-\mathrm{CO}$; however, they point out that it is $\pi_{2}$ - CO (recall Observation 2.7). Hence, they apply the SOR theory to this frequency domain matrix partitioned as a block $2 \times 2$ matrix.

O'Leary [15] considered ordering schemes that would allow for the efficient implementation on parallel computers of SOR-type iterative processes. These schemes include the " $P^{3}$," " $T^{3}, "$ " $H+H$," "Cross," and "Box" orderings; the names are indicative of the patterns made by the blocks of grid points of a single color, and each ordering uses three colors. These ordering schemes give rise to coefficient matrices of the form (2), where each of the diagonal blocks $A_{i i}$ is not a diagonal matrix. For example, partitioned as a block $3 \times 3$ matrix, the coefficient matrix corresponding to a $P^{3}$ ordering of the grid points (under a nine-point stencil) has diagonal blocks $A_{i i}$ which are block diagonal. O'Leary gives the sparsity structure for this matrix, and it is immediately apparent that it is not $\pi_{3}-\mathrm{CO}$ by Theorem 3.1 since blocks $A_{1,2}$ and $A_{2,3}$ contain nonzero entries but so does block $A_{1,3}$. The grid corresponding to the sparsity structure pictured in [15] has five points "per $P$ " if the block of points $P$ is internal to the grid. Thus many of the diagonal blocks internal to the $A_{i i}, i=1,2,3$, are $5 \times 5$ and full. Therefore, this matrix is also not CO (see Observation 2.6).

Shortley and Weller [19] considered the use of $k \times k$ square blocks of points with the Gauss-Seidel method for the solution of (31). Parter and Steuerwalt [17] point out that $k \times k$ block orderings lead to coefficient matrices which satisfy block property $A$ (Young [25] refers to this as property $A^{(\pi)}$ ); in fact, these orderings give rise to $\pi$-CO matrices. We obtain a coefficient matrix of the form $T_{p}+B_{r}$ where $T_{p}$ is a block $\left(\frac{N}{k}\right)^{2} \times\left(\frac{N}{k}\right)^{2}$ tridiagonal matrix of the form (6), (7) with zero blocks every $\frac{N}{k}$ blocks on the first sub- and superdiagonals. $B_{r}$ is block $\frac{N}{k} \times \frac{N}{k}$ bidiagonal of the form (15) with nonzero blocks $B_{k, k+1}^{m_{k}}$ which are block $\frac{N}{k} \times \frac{N}{k}$ unidiagonal with $m_{k}=m_{L}=1$. Thus the coefficient matrix corresponding to a $k \times k$ block ordering on an $N \times N$ grid is $\pi_{(N / k)^{2}}-\mathrm{CO}$ by Theorem 3.5.

Duff and Meurant [4] considered preconditioning by incomplete factorization in 17 different orderings, including the natural, red/black, zebra, and four-color orderings already discussed. The methods of this paper can be applied to many of the orderings discussed there including forward, reverse, and alternating diagonal orderings (Young showed that a forward diagonal ordering gives a CO matrix), a diagonal ordering of $k \times k$ blocks, a spiral ordering, and two block orderings attributed to Van der Vorst; see Harrar [8].

Although the Laplace problem with Dirichlet boundary conditions (BCs) yields a $\mathrm{CO}$ system of equations under a natural ordering, if we assume periodic BCs in either coordinate direction, the coefficient matrix is no longer $\mathrm{CO}$. The theory of $\S 4$ indicates that it is also no longer $\pi_{N}$-CO. However, now consider a red/black ordering. With an even number $N$ of grid points in each direction, the matrix is block $2 \times 2$ with blocks of dimension $N^{2} / 2$. It is trivially $\pi_{2}$-CO (Observation 2.7), and its diagonal blocks are diagonal matrices; thus it is also $\mathrm{CO}$ by Theorem 3.7. We note that with an odd number of grid points in either direction, the matrix is not, in general, CO under a red/black ordering since we would no longer have full decoupling. Boundary unknowns would depend on unknowns of the same color interior to the grid so that the diagonal blocks of the coefficient matrix would no longer be diagonal. One way to overcome this difficulty may be to consider certain coloring schemes with more than two colors; see Harrar [7]. 
6. $T_{p}(q, r)$-matrices and $(q, r)$-CO matrices. As pointed out in $\S 1$, there are several generalizations of the class of CO matrices other than that of the class of $\pi$-CO matrices. These include $(q, r)-C O$, generalized $(q, r)-C O((q, r)-G C O)$, and $\pi-G C O$ matrices (Young [25]). In this section, we do not treat either of these "generalized" versions, although we try to give a few examples of the ways in which our previous results can be used to obtain some information concerning $(q, r)$ - $\mathrm{CO}$ matrices. In particular, these results apply to the class of $T_{p}(q, r)$-matrices; this class represents a generalization of the class of $T$-matrices originally defined in $\S 2$. We note that in this section, $q$ and $r$ have no relation to the $q$ and $r$ of previous sections. When we mean $q$ and $r$ as used previously, we denote them by $\hat{q}$ and $\hat{r}$.

A formal definition of a $(q, r)$-CO matrix can be found in Young [25]. We note only that a $(1,1)$-CO matrix is a CO matrix in the sense of $\S 2$. Analogous to this generalization of $\mathrm{CO}$ matrices, we generalize the concept of a $T_{p}$-matrix to obtain Definition 6.1 (Young [25]).

Definition 6.1. Let $q$ and $r$ be positive integers less than $p$. The matrix $A$ is a $T_{p}(q, r)$-matrix if it can be partitioned into the block $p \times p$ form $A=\left(A_{i j}\right)$ where, for each $i, A_{i i}=D_{i}$ is a square diagonal matrix and where all other blocks vanish, except possibly for the blocks $A_{i, i+r}, i=1,2, \ldots, p-r$ and $A_{i, i-q}, i=q+1, q+2, \ldots, p$.

Clearly a $T_{p}(1,1)$-matrix is a $T_{p}$-matrix as given by Definition 2.8. Also, just as $T$-matrices are CO, so too are $T(q, r)$-matrices $(q, r)$-CO. However, we try to show under what circumstances $T_{p}(q, r)$-matrices are also $\pi_{p}$-CO and, since their diagonal blocks are diagonal matrices, $\mathrm{CO}$ by Theorem 3.7 .

Now, note that for the purposes of showing $(\pi-)$ consistent ordering, a $T_{p}(q, r)$ matrix with either $q=1$ or $r=1$ can be treated as a matrix of the form $T_{p}+B_{\hat{r}}$ where $T_{p}$ is a $T_{p}$-matrix and $B_{\hat{r}}$ is a block $\hat{r} \times \hat{r}$ bidiagonal matrix with nonzero elements on its $q$ th or $r$ th sub- or superdiagonal, respectively. The case $q=1$ is of particular importance since Varga [22] gave a complete analysis of this case, and then Nichols and Fox [14] showed that the SOR method is not effective if $q>1$. Also, the important class of $p$-cyclic matrices, $p \geq 2$ consists of matrices with nonzero diagonal elements which have a corresponding Jacobi iteration matrix that is permutationally similar to a $T_{p}(1, r)$-matrix where $r=p-1$. Therefore, in what follows, we consider only the case in which one of $q$ and $r$ is unity.

Appealing to Theorem 3.1, we obtain our first result.

THEOREM 6.2. Let $q$ and $r$ be positive integers less than $p$. Suppose $A$ is a $T_{p}(1, r)$-matrix such that $A_{i, i+r} \neq 0, i=1, \ldots, p-r$ and $A_{i, i-1} \neq 0, i=2, \ldots, p$. Similarly, suppose $\hat{A}$ is a $T_{p}(q, 1)$-matrix such that $\hat{A}_{i, i+1} \neq 0, i=1, \ldots, p-1$ and $\hat{A}_{i, i-q} \neq 0, i=q+1, q+2, \ldots, p$. Then $A$ is $\pi_{p}-C O$ and $C O$ if and only if $r=1$, and $\hat{A}$ is $\pi_{p}-C O$ and $C O$ if and only if $q=1$.

Proof. Let $A(\hat{A})$ be as given in the hypothesis of the theorem. Then $A(\hat{A})$ has no zero blocks on its first subdiagonal (superdiagonal). Suppose that $A(\hat{A})$ is $\pi_{p}$-CO. Then, by Theorem 3.1, $A(\hat{A})$ can have no zero blocks outside the first off-diagonals. That is, we must have $r=1(q=1)$.

Now, assume that $r=1(q=1)$. Then $A(\hat{A})$ is a $T_{p}(1,1)$-matrix, i.e., a $T_{p^{-}}$ matrix. Thus, by Theorem $2.9, A(\hat{A})$ is $\pi_{p}$ CO, and, by Theorem $3.7, A(\hat{A})$ is CO.

Analogous to our progression in $\S 3$, we now consider the case in which the first subdiagonal $(q=1)$ or the first superdiagonal $(r=1)$ has some zero blocks. The remainder of the results of this section are stated only for the case $q=1$, but it is trivial to adjust the proofs to handle the case $r=1$; this should be clear from the 
proof of Theorem 6.2 given above.

First, we treat the case in which $A$ is a $T_{p}(1, r)$-matrix, with $r=p-1$, and has at least one zero block on the $q=1$ subdiagonal; in this case, $A$ is trivially a $p$-cyclic matrix.

TheOREM 6.3. Let $A$ be $a T_{p}(1, p-1)$-matrix, $p>2 . A$ is $\pi_{p}-C O$ and $C O$ if and only if $A_{i, i-1}=0$ for some $i=2, \ldots, p$.

Proof. Let $A$ be a $\pi_{p}$-CO $T_{p}(1, p-1)$-matrix and assume that $A_{i, i-1} \neq 0$ for $i=2, \ldots, p$. By Theorem 6.2, a $T_{p}(1, r)$-matrix, all of whose blocks on the first subdiagonal are nonzero, can be $\pi_{p}$-CO if and only if $r=1$. However, we have $r=p-1$, a contradiction. Thus we must have that one of $A_{i, i-1}, i=2, \ldots, p$ is zero.

Now, assume that $A_{k, k-1}=0$ where $k=2, \ldots, p$ is fixed. The nonzero blocks of $A$ are $A_{1, p}$ and $A_{i, i-1}, i=2, \ldots, k-1, k+1, \ldots, p$. Thus, in the construction of a $\pi_{p}$-compatible ordering vector for $A$, we require $\gamma_{p}-\gamma_{1}=1$ and $\gamma_{i}-\gamma_{i-1}=1$, where $i=2, \ldots, k-1, k+1, \ldots, p$. We may easily verify that the elements of the vector

$$
\gamma^{\left(\pi_{p}\right)}=(p, p+1, \ldots, p+k-2, k+2, k+3, \ldots, p+1)^{T}
$$

satisfy both of these requirements. Thus $A$ is $\pi_{p}$-CO by Theorem 2.5 and CO by Theorem 3.7.

In $\S 3.2$ we considered the case in which $T_{p}$ was tridiagonal with intermittent zeros every $\hat{q}$ th entry on the first sub- and superdiagonal. We conclude this section with a natural extension of the results of that section. Recall that there we eventually allowed $m_{k}$, which determined the position of the sole nonzero block diagonal in the block $B_{k, k+1}^{m_{k}}$ of $B_{\hat{r}}$, to vary with each block. However, here we must keep $m_{k}$ constant for all $k$ so that the nonzero blocks $A_{l+i, k q+j}$ of all of the $B_{k, k+1}$ will lie along the same diagonal. This leads us to the final result of this section, which we state without proof.

TheOREM 6.4. Let $A$ be a $T_{p}(1, r)$-matrix with zero blocks every $\hat{q}$ th position on the first $(q=1)$ subdiagonal. Suppose that $r=\hat{q}+m_{U}$ for some $m_{U}=2, \ldots, \hat{q}$ or $r=\hat{q}-\left(m_{L}-2\right)$ for some $m_{L}=1, \ldots, \hat{q}$. If the rth superdiagonal has $m-1$ zero blocks following every $\hat{q}-(m-1)$ possibly nonzero blocks (where $m=m_{U}$ or $m=m_{L}$, depending on whether $r=r\left(m_{U}\right)$ or $r=r\left(m_{L}\right)$, respectively), then $A$ is $\pi_{p}-C O$ and CO.

7. Summary. Multicoloring provides a valuable technique to increase efficiency in implementing many iterative processes (SOR-type, PCG, multigrid, etc.) to solve linear systems of equations, especially on today's parallel and vector computers.

Application of Young's classical SOR theory is valid when the coefficient matrix of the system to be solved is CO. Often, especially when a multicoloring scheme is introduced, we obtain a coefficient matrix that is not $\mathrm{CO}$; however, this matrix may be $\pi$-CO (block $\mathrm{CO}$ ) for some partitioning $\pi$, although determination of whether or not a given matrix is $\mathrm{CO}$ or $\pi$-CO is generally nontrivial. Though computer programs exist to determine consistent ordering (Young [25]), these may be impractical for very large matrices and do not, in general, take into account the sparsity structure inherent in the coefficient matrices corresponding to multicolored systems. We have presented some theory which allows us to ascertain quickly whether matrices which have an underlying block tridiagonal structure are $(\pi-) \mathrm{CO}$ or not; such matrices are often obtained when a multicoloring scheme is used.

We applied the theory to ordering schemes from the literature to show that while some commonly used orderings give rise to $\mathrm{CO}$ or $\pi_{p}$ - $\mathrm{CO}(p>2)$ matrices, many 
others do not. This is particulary true for multicolor orderings with more than two colors.

Acknowledgments. The author would like to thank Professors Herbert B. Keller, James M. Ortega, and David M. Young for looking over preliminary versions of this manuscript and Douglas A. Waetjen for helpful discussions. The detailed comments of two of the referees (one of whom pointed out the similarity between Theorem 3.7 and Theorem 14.3.2 of Young [25]) are also greatly appreciated.

\section{REFERENCES}

[1] L. Adams and H. Jordan, Is SOR color-blind?, SIAM J. Sci. Statist. Comput., 7 (1986), pp. $490-506$.

[2] L. Adams ANd J. Ortega, A multi-color SOR method for parallel computation, in Proc. 1982 Internat. Conf. Parallel Processing, Bellaire, MI, 1982, pp. 53-58.

[3] L. ChONG AND D.-Y. CAI, Relationship between eigenvalues of Jacobi and SSOR iteration matrices for weakly p-cyclic matrix, J. Comput. Math. Coll. Univ., 1 (1985), pp. 79-84. (In Chinese.)

[4] I. DUFF AND G. MEURANT, The effect of ordering on preconditioned conjugate gradients, BIT, 29 (1989), pp. 635-658.

[5] H. Foerster, K. StüBen, And U. Trottenberg, Non-standard multigrid techniques using checkered relaxation and intermediate grids, in Elliptic Problem Solvers, M. Schultz, ed., Academic Press, New York, 1981, pp. 285-300.

[6] L. Hageman and D. Young, Applied Iterative Methods, Academic Press, New York, 1981.

[7] D. HARRAR II, Multicolor orderings for the concurrent iterative solution of non-Dirichlet problems, manuscript.

[8] - Alternative orderings, multicoloring schemes, and consistently ordered matrices, Tech. Rep. CRPC-90-8, Dept. of Applied Mathematics, California Institute of Technology, Pasadena, CA, 1990.

[9] D. Harrar II And J. Ortega, Optimum m-step SSOR preconditioning, J. Comput. Appl. Math., 24 (1988), pp. 195-198.

[10] - Multicoloring with lots of colors, in Proc. Third Internat. Conf. Supercomput., Crete, Greece, 1989, pp. 1-6.

[11] — Solution of three-dimensional generalized Poisson equations on vector computers, in Iterative Methods for Large Linear Systems, Academic Press, New York, 1990.

[12] C.-C. Kuo AND B. Levy, A two-level four-color SOR method, SIAM J. Numer. Anal., 26 (1989), pp. 129-151.

[13] X. LI AND R. VARGA, A note on the SSOR and USSOR iterative methods applied to p-cyclic matrices, in Iterative Methods for Large Linear Systems, Academic Press, New York, 1990.

[14] N. Nichols AND L. Fox, Generalized consistent ordering and optimum successive overrelaxation factor, Numer. Math., 13 (1969), pp. 425-433.

[15] D. O'LEARY, Ordering schemes for parallel processing of certain mesh problems, SIAM J. Sci. Statist. Comput., 6 (1984), pp. 761-770.

[16] J. ORTEGA AND R. VOIGT, Solution of partial differential equations on vector and parallel computers, SIAM Rev., 27 (1985), pp. 149-240.

[17] S. Parter And M. Steuerwalt, On $K$-line and $K \times K$ block iterative schemes for a problem arising in three-dimensional elliptic difference equations, SIAM J. Numer. Anal., 17 (1980), pp. 823-839.

[18] E. Poole And J. Ortega, Multicolor ICCG methods for vector computers, SIAM J. Numer. Anal., 24 (1987), pp. 1394-1418.

[19] G. Shortley AND R. Weller, The numerical solution of Laplace's equation, J. Appl. Phys., 4 (1938), pp. 334-348.

[20] K. STüBen AND U. Trottenberg, Multigrid methods: Fundamental algorithms, model problem analysis, and applications, in Multigid Methods, Proceedings, Koln-Porz, 1981, W. Hackbush and U. Trottenberg, eds., Springer-Verlag, Berlin, New York, 1982, pp. 1176.

[21] R. VARGA, Orderings of the successive overrelaxation scheme, Pacific J. Math., 9 (1959), pp. 925-939.

[22] —, p-cyclic matrices: A generalization of the Young-Frankel successive overrelaxation scheme, Pacific J. Math., 9 (1959), pp. 617-628. 
[23] R. VARgA, Matrix Iterative Analysis, Prentice-Hall, Englewood Cliffs, NJ, 1962.

[24] D. Young, Iterative Methods for Solving Partial Differential Equations of Elliptic Type, Ph.D. thesis, Harvard University, Cambridge, MA, 1950.

[25] - Iterative Solution of Large Linear Systems, Academic Press, New York, 1971. 